\title{
New insights into the spin structure of the nucleon
}

\author{
Véronique Bernard*\#1, Evgeny Epelbaum#2, Hermann Krebs $\star^{\# 3}$, Ulf-G. Meißner ${ }^{\ddagger \pm 4}$ \\ * Groupe de Physique Théorique, Institut de Physique Nucléaire \\ CNRS/Université Paris-Sud 11, 91406 Orsay Cedex, France \\ *Institut für Theoretische Physik II, Ruhr-Universität Bochum, \\ D-44780 Bochum, Germany \\ $\ddagger$ Universität Bonn, Helmholtz-Institut für Strahlen- und Kernphysik (Theorie) and \\ Bethe Center for Theoretical Physics, Universität Bonn, D-53115 Bonn, Germany \\ ${ }^{\dagger}$ Forschungszentrum Jülich, Institut für Kernphysik, Institute for Advanced Simulation \\ and Jülich Center for Hadron Physics, D-52425 Jülich, Germany
}

\begin{abstract}
We analyze the low-energy spin structure of the nucleon in a covariant effective field theory with explicit spin-3/2 degrees of freedom to third order in the small scale expansion. Using the available data on the strong and electromagnetic width of the $\Delta$-resonance, we give parameter-free predictions for various spin-polarizabilities and moments of spin structure functions. We find an improved description of the nucleon spin structure at finite photon virtualities for some observables and point out the necessity of a fourth order calculation.
\end{abstract}

Keywords: double virtual Compton scattering, nucleon spin structure, effective field theory PACS: $12.39 . \mathrm{Fe}, 11.55 . \mathrm{Fv}, 14.20 . \mathrm{Dh}$

\footnotetext{
\#1 email: bernard@ipno.in2p3.fr

\#2 email: evgeny.epelbaum@rub.de

\#3 email: hermann.krebs@rub.de

\#4 email: meissner@hiskp.uni-bonn.de
} 


\section{Introduction}

The internal spin structure of the nucleon which is described by structure functions in deep inelastic lepton-hadron scattering has been extensively studied in the last decades both theoretically and experimentally, see e.g. [1] for a review. The structure functions are related to the real, virtual or double virtual Compton scattering $\left(\mathrm{V}^{2} \mathrm{CS}\right)$ amplitudes by various sum rules which connect information at all energy scales. On the experimental side only recently it has become possible to work with polarized beams and polarized targets which is necessary for studying the nucleon spin structure. One of the main goals of the Jefferson Lab activities is to provide a precise experimental mapping of spin-dependent observables from low momentum transfer to the multi-GeV region, see e.g. [2 4] for the early measurements (that also cover the low-energy region). Concerning the low-energy spin structure - which is at the center of this investigation - more data also at smaller photon virtualities $Q^{2}$ have been taken at Jefferson Lab and their analysis will be completed soon, see e.g. Refs. [5, 6]. Therefore, it is timely to reconsider the theoretical predictions for the moments of the nucleon spin structure functions.

At very low energies, far below the chiral symmetry scale of the order of $1 \mathrm{GeV}$, the nucleon dynamics is dominated by chiral symmetry of QCD and for this reason can be rigorously described by chiral perturbation theory (CHPT). This theory provides a systematic expansion in low momenta and masses of the Goldstone bosons (identified with the pions in the case of two flavors). At low photon virtualities one can make rigorous predictions for the spin-dependent part of $\mathrm{V}^{2} \mathrm{CS}$ and use the Jefferson Lab data to test the chiral dynamics of QCD.

$\mathrm{V}^{2} \mathrm{CS}$ has already been considered within the CHPT framework up to $\mathcal{O}\left(q^{4}\right)$ in the chiral expansion by several theoretical groups, see [7] for a review (here, $q$ denotes a genuine small parameter like external momenta or the pion mass). In standard CHPT, all effects of the $\Delta(1232)$-resonance degrees of freedom are encoded in the low-energy constants. Chiral symmetry prevents spin-dependent counterterms of $\mathcal{O}\left(q^{3}\right)$ and $\mathcal{O}\left(q^{4}\right)$ in $\mathrm{V}^{2} \mathrm{CS}$ such that their first contribution is possible at $\mathcal{O}\left(q^{5}\right)$ in this scheme. However, once one introduces the spin-3/2 degrees of freedom explicitly, one realizes that their tree-level contributions are large and far from being suppressed. This suggests to perform a systematic analysis of $\mathrm{V}^{2} \mathrm{CS}$ with explicit deltas by using a covariant version of the so-called small scale expansion (SSE) [13]. In that extension of CHPT, the nucleon-delta mass splitting, $\Delta=m_{\Delta}-m_{N}$, is counted as an additional small parameter, thus the generic small parameter $\varepsilon$ collects external momenta, the pion mass and $\Delta$. Note that calculations within the SSE employing the heavy-baryon (HB) expansion were already performed by Kao et al. [8]. Here, we address this issue up to the order $\varepsilon^{3}$ in a covariant SSE. In contrast to the the covariant CHPT calculation of Bernard et al. [9, 10, we do not use the method of infrared regularization here as it leads to deformations of the analytical structure at higher virtualities that leave a trace in the $Q^{2}$-dependence of certain observables (see also Ref. [11] for an early study of $\gamma_{0}\left(Q^{2}\right)$ using a relativistic version of baryon CHPT). We obtain parameter-free predictions for various moments of the spin structure functions at low virtualities. We focus, in particular, on the so-called forward and longitudinal-transverse spin-polarizabilities, as these have posed particular problems to the CHPT calculations. More precisely, the proton spin polarizability at the photon point comes out larger in magnitude than experiment for most calculations and also the magnitude of the $Q^{2}$-dependent neutron longitudinal-transverse spin polarizability is predicted much smaller than found in experiment. This latter finding is particularly puzzling as the leading $\Delta$-resonance contributions are expected to cancel here. In this paper, we will shed new light on these issues. It is also important to stress that the contributions considered here are nothing but the leading order terms in the chiral expansion of these spin polarizabilities based on an effective Lagrangian of pions, nucleons, deltas and photons.

Our manuscript is organized as follows: In Sec. 2, we give the necessary formalism for double virtual 
Compton scattering off the nucleon and the definition of the pertinent moments of spin structure

functions that are amenable to a low-energy expansion. Sec. 3 is devoted to a short discussion of the underlying effective Lagrangian, the covariant treatment of the baryon fields and the pertinent Feynman diagrams to be calculated. Our results are discussed and presented in Sec. 4. We end with a short summary and outlook in Sec. 5 .

\section{Formalism I: Double virtual Compton scattering}

The forward tensor for double virtual Compton scattering in terms of the electromagnetic current $J^{\mu}$ is given by

$$
\begin{aligned}
T^{[\mu \nu]} & =i \int d^{4} x e^{i q \cdot x}\left\langle P S\left|T J^{\mu}(x) J^{\nu}(0)\right| P S\right\rangle \\
J^{\mu}(x) & =\sum_{i} e_{i} \bar{\psi}_{i}(x) \gamma^{\mu} \psi_{i}(x)
\end{aligned}
$$

Here, $\psi_{i}(x)$ denotes a quark field of flavor $i$ with charge $e_{i}$, while $P$ and $S$ are the momentum and spin polarization of the nucleon, respectively. The spin-dependent $\mathrm{V}^{2} \mathrm{CS}$ tensor can be parameterized by two structure functions

$$
T^{[\mu \nu]}=-\frac{i}{2} \epsilon^{\mu \nu \alpha \beta} q_{\alpha}\left[S_{\beta} S_{1}\left(\nu, Q^{2}\right)+\frac{1}{m_{N}^{2}}\left(P \cdot q S_{\beta}-S \cdot q P_{\beta}\right) S_{2}\left(\nu, Q^{2}\right)\right],
$$

which depend on two independent scalar variables $Q^{2}=-q^{2}$ (the photon virtuality) and $\nu=P \cdot q / m_{N}$ (the photon energy), where $m_{N}$ is the nucleon mass. On the other hand, the differential cross section of polarized spin-dependent inclusive lepton-nucleon scattering (in the one-photon-exchange approximation) is proportional to the antisymmetric tensor

$$
W^{[\mu \nu]}=\frac{1}{4 \pi} \int d^{4} x e^{i q \cdot x}\left\langle P S\left|\left[J^{\mu}(x), J^{\nu}(0)\right]\right| P S\right\rangle
$$

which can be parameterized again by two structure functions

$$
W^{[\mu \nu]}=-\frac{i}{2} \epsilon^{\mu \nu \alpha \beta} q_{\alpha}\left[S_{\beta} G_{1}\left(\nu, Q^{2}\right)+\frac{1}{m_{N}^{2}}\left(P \cdot q S_{\beta}-S \cdot q P_{\beta}\right) G_{2}\left(\nu, Q^{2}\right)\right] .
$$

The amplitudes for $\mathrm{V}^{2} \mathrm{CS}$ can be analytically continued to the complex $\nu$-plane. They have poles at $\nu_{c}= \pm Q^{2} / 2 m_{N}$, corresponding to $s$ - and $u$-channel elastic scattering and two cuts on the real axis extending from $\nu= \pm \nu_{c}$ to $\pm \infty$. Using analyticity and assuming a sufficient fast fall-off of the structure functions at large $\nu$, one can relate the corresponding structure functions to each other by dispersion integrals 12

$$
\begin{aligned}
& S_{1}\left(\nu, Q^{2}\right)=4 \int_{Q^{2} / 2 m_{N}}^{\infty} \frac{d z z G_{1}\left(z, Q^{2}\right)}{z^{2}-\nu^{2}} \\
& S_{2}\left(\nu, Q^{2}\right)=4 \int_{Q^{2} / 2 m_{N}}^{\infty} \frac{d z \nu G_{2}\left(z, Q^{2}\right)}{z^{2}-\nu^{2}} .
\end{aligned}
$$

In the derivation of these relations crossing symmetry

$$
S_{1}\left(-\nu, Q^{2}\right)=S_{1}\left(\nu, Q^{2}\right), \quad S_{2}\left(-\nu, Q^{2}\right)=-S_{2}\left(\nu, Q^{2}\right),
$$


has been used. Usually one works with the inelastic version of the dispersion relations where by inelastic we mean that the elastic part of the amplitudes - which have a pole at $Q^{2} / 2 m_{N}$ - is subtracted. In this case the cut starts at the pion production threshold $s=s_{0}=\left(m_{N}+M_{\pi}\right)^{2}$, which is equivalent to $\nu_{0}=\left(Q^{2}+M_{\pi}^{2}\right) /\left(2 m_{N}\right)+M_{\pi}$ The dispersion relations are then given by

$$
\begin{aligned}
& \bar{S}_{1}\left(\nu, Q^{2}\right)=4 \int_{\nu_{0}}^{\infty} \frac{d z z G_{1}\left(z, Q^{2}\right)}{z^{2}-\nu^{2}}, \\
& \bar{S}_{2}\left(\nu, Q^{2}\right)=4 \int_{\nu_{0}}^{\infty} \frac{d z \nu G_{2}\left(z, Q^{2}\right)}{z^{2}-\nu^{2}}
\end{aligned}
$$

with $\bar{S}_{i}\left(\nu, Q^{2}\right)=S_{i}\left(\nu, Q^{2}\right)-S_{i}^{\text {elastic }}\left(\nu, Q^{2}\right)$

For small photon energies, the $\mathrm{V}^{2} \mathrm{CS}$ amplitudes can be expanded in powers of $\nu^{2}$ :

$$
\begin{aligned}
& \bar{S}_{1}\left(\nu, Q^{2}\right)=\sum_{i=0}^{\infty} \bar{S}_{1}^{(2 i)}\left(0, Q^{2}\right) \nu^{2 i} \\
& \bar{S}_{2}\left(\nu, Q^{2}\right)=\sum_{i=0}^{\infty} \bar{S}_{2}^{(2 i+1)}\left(0, Q^{2}\right) \nu^{2 i+1}
\end{aligned}
$$

The next-to-leading order coefficients (called the forward and the longitudinal-transverse spin-polarizability, respectively) in this series are directly related to the moments of structure functions and can be measured. In this work, we concentrate on the low-energy region. The aforementioned forward and longitudinal-transverse polarizabilities are given by

$$
\begin{aligned}
& \gamma_{0}\left(Q^{2}\right)=\frac{1}{8 \pi}\left(\bar{S}_{1}^{(2)}\left(0, Q^{2}\right)-\frac{Q^{2}}{m_{N}} \bar{S}_{2}^{(3)}\left(0, Q^{2}\right)\right) \\
& \delta_{0}\left(Q^{2}\right)=\frac{1}{8 \pi}\left(\bar{S}_{1}^{(2)}\left(0, Q^{2}\right)+\frac{1}{m_{N}} \bar{S}_{2}^{(1)}\left(0, Q^{2}\right)\right) .
\end{aligned}
$$

They obviously can be described as dispersion integrals and can be rigorously calculated by CHPT at low virtualities. Using a dispersion representation one has access to experimental data such that polarizabilities provide a testing ground for chiral dynamics of QCD. Similar formulae can be given for the generalized GDH integral $I_{A}\left(Q^{2}\right)$ and the first moments of the spin structure functions $\Gamma_{1}\left(Q^{2}\right)$, see Ref. [12]. For completeness, we give the corresponding expressions:

$$
\begin{array}{r}
I_{A}\left(Q^{2}\right)=\frac{m_{N}^{2}}{4 e^{2}}\left[\bar{S}_{1}^{(0)}\left(0, Q^{2}\right)-\frac{Q^{2}}{m_{N}} \bar{S}_{2}^{(1)}\left(0, Q^{2}\right)\right], \\
\Gamma_{1}\left(Q^{2}\right)=\frac{Q^{2}}{2 m_{N}^{2}} I_{1}\left(Q^{2}\right), \quad I_{1}\left(Q^{2}\right)=\frac{m_{N}^{2}}{4 e^{2}} \bar{S}_{1}^{(0)}\left(0, Q^{2}\right) .
\end{array}
$$

These observables will also be considered here.

\section{Formalism II: Effective Lagrangian and one-loop calculation}

We now consider the underlying chiral Lagrangian. In Ref. [10], the chiral pion-nucleon Lagrangian in the presence of external sources, $\mathcal{L}_{\pi N}\left[U, N, \bar{N} ; s, p, v_{\mu}, a_{\mu}\right]$, was utilized combined with infrared regularization to separate the soft (long-range) from the hard (short distance) dynamics. Contributions from the $\Delta(1232)$ resonance at tree level were added in a phenomenological approach and shown to be important. Here, we improve this calculation by extending the underlying effective field theory 

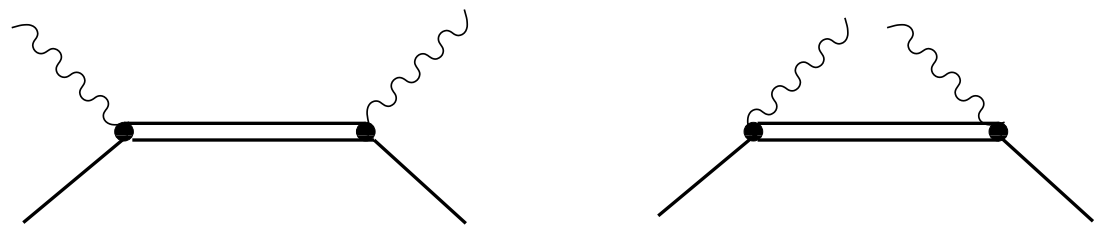

Figure 1: Tree diagrams at $\mathcal{O}\left(\varepsilon^{3}\right)$. Solid, double and wiggly lines denote nucleons, deltas and photons, in order. The filled circle is an insertion from $\mathcal{L}_{\pi N \Delta}^{(2)}$.

(EFT) to include the delta based on the so-called covariant small scale expansion to $\mathcal{O}\left(\varepsilon^{3}\right)$. We use the explicit form of the spin-3/2 propagator from Ref. [14. Also, we do not use infrared regularization as done in Ref. [10] but rather utilize dimensional regularization. For the case at hand, this is a consistent scheme as counter terms in the four-point function only show up at $\mathcal{O}\left(\varepsilon^{5}\right)$. Therefore, no power-counting violating contributions appear up-to-and-including $\mathcal{O}\left(\varepsilon^{4}\right)$ and the corresponding loop corrections to $\mathrm{V}^{2} \mathrm{CS}$ are all finite after mass and coupling constant renormalization. Note, however, that one has to deal with some LECs in the three-point functions that appear as parts of the fourth order diagrams. In case of nucleon intermediate states, these are nothing but the anomalous magnetic moment of the proton and the neutron, see also Ref. [10]. In case of delta intermediate states, we have in addition dimension two LECs from $\mathcal{L}_{\pi N \Delta}^{(3)}$, that can be fixed from $\Delta \rightarrow N$ transition form factors.

The chiral Lagrangian for $\mathrm{V}^{2} \mathrm{CS}$ in the pion-nucleon sector is given in Ref. [10]. The pertinent new Lagrangian structures related to the inclusion of the spin-3/2 fields read (for the construction principles, see [13])

$$
\begin{aligned}
\mathcal{L}_{\pi N \Delta}^{(1)} & =h_{A} \bar{\psi}_{i}^{\mu} \omega_{\mu}^{i} N+\text { h.c. } \\
\mathcal{L}_{\pi \Delta \Delta}^{(1)} & =\bar{\psi}_{i}^{\mu}\left(i \not D_{\mu \nu}^{i j}-m_{\Delta} \gamma_{\mu \nu} \delta^{i j}\right) \psi_{j}^{\nu} \\
\mathcal{L}_{\pi N \Delta}^{(2)} & =\frac{1}{2} b_{1} \bar{\psi}_{i}^{\mu} i f_{+\mu \alpha}^{i} \gamma^{\alpha} \gamma_{5} N+\text { h.c. }
\end{aligned}
$$

with

$$
\begin{aligned}
\not D_{\mu \nu}^{i j} & =\gamma_{\mu \nu \alpha} D_{i j}^{\alpha}, \quad D_{i j}^{\alpha}=\left(\partial^{\alpha}+\Gamma^{\alpha}\right) \delta_{i j}-i \epsilon_{i j k}\left\langle\tau^{k} \Gamma^{\alpha}\right\rangle \\
\Gamma^{\alpha} & =\frac{1}{2}\left[u^{\dagger}, \partial_{\mu} u\right]-\frac{i}{2} u^{\dagger}\left(v_{\mu}+a_{\mu}\right) u-\frac{i}{2} u\left(v_{\mu}-a_{\mu}\right) u^{\dagger} \\
f_{+\mu \alpha}^{i} & =\frac{1}{2}\left\langle\tau^{i} f_{+\mu \nu}\right\rangle, \quad \omega_{\mu}^{i}=\frac{1}{2}\left\langle\tau^{i} u_{\mu}\right\rangle, \\
\gamma_{\mu \nu \alpha} & =\frac{1}{4}\left\{\left[\gamma_{\mu}, \gamma_{\nu}\right], \gamma_{\alpha}\right\}, \quad \gamma_{\mu \nu}=\frac{1}{2}\left[\gamma_{\mu}, \gamma_{\nu}\right] .
\end{aligned}
$$

Here, $\psi_{i}^{\mu}$ is a conventional Rarita-Schwinger spinor for the spin-3/2 fields, $N$ denotes the nucleon bispinor (throughout, we work in the isospin limit), $h_{A}$ is the leading $\pi N \Delta$ axial-coupling (analogous to $g_{A}$ in the pion-nucleon sector) and $b_{1}$ is the leading photon-nucleon-delta coupling of chiral dimension two (much like the nucleon magnetic moment that appears first in $\mathcal{L}_{\pi N}^{(2)}$ ). As usual, the pions are collected in the matrix-valued field $U(x)=u^{2}(x)$. We only need the external vector source $v_{\mu}=Q A_{\mu}$, with $A_{\mu}$ the photon field and $Q=(1,0) e$ the nucleon charge matrix. Therefore $f_{+\mu \nu}=F_{\mu \nu}\left(u Q u^{\dagger}+\right.$ $\left.u^{\dagger} Q u\right)$.

Based on this, we are now in the position to calculate $\mathrm{V}^{2} \mathrm{CS}$ at low energies in the covariant SSE. At order $\mathcal{O}\left(\varepsilon^{3}\right)$, we have tree and the leading one-loop graphs involving the $\Delta$-resonance, see Figs. 1 and 2, respectively. The corresponding third order pion-nucleon loop graphs are e.g. displayed 

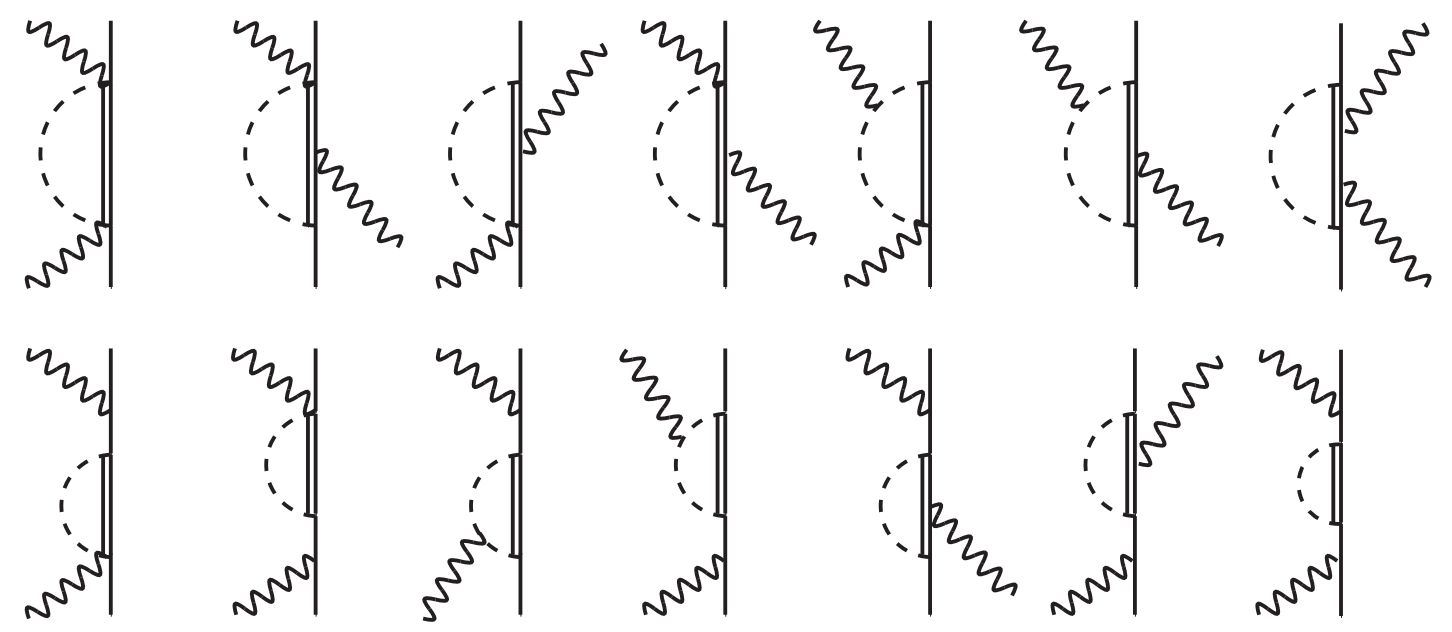

Figure 2: Delta-loop diagrams at $\mathcal{O}\left(\varepsilon^{3}\right)$. Solid, double, dashed and wiggly lines denote nucleons, deltas, pions and photons, in order. Crossed graphs are not shown.

in Fig. 1 of Ref. [10]. Note that since the delta propagator is of $\mathcal{O}\left(\varepsilon^{-1}\right)$, the tree graphs with two insertions from $\mathcal{L}_{\pi N \Delta}^{(2)}$ appear first at third order in the SSE. Note further that at this order there are no unknown low-energy constants (LECs), since the couplings $h_{A}$ and $b_{1}$ can be determined from the decays $\Delta \rightarrow N \pi$ and $\Delta \rightarrow N \gamma$, respectively. More precisely, the strong width of the $\Delta$ is given in terms of the LEC $h_{A}$ as

$$
\Gamma_{\Delta}^{\mathrm{str}}=h_{A}^{2} \frac{\left(\left(m_{\Delta}-m_{N}\right)^{2}-M_{\pi}^{2}\right)^{3 / 2}\left(\left(m_{\Delta}+m_{N}\right)^{2}-M_{\pi}^{2}\right)^{5 / 2}}{192 F_{\pi}^{2} \pi m_{\Delta}^{5}}=(118 \pm 2) \mathrm{MeV}
$$

and similarly for the electromagnetic width in terms of $b_{1}$

$$
\Gamma_{\Delta}^{\mathrm{em}}=e^{2} b_{1}^{2} \frac{\left(m_{\Delta}^{2}-m_{N}^{2}\right)^{3}\left(3 m_{\Delta}^{2}+m^{2}\right)}{576 \pi m_{\Delta}^{5}},
$$

with $\Gamma_{\Delta}^{\mathrm{em}} /\left(\Gamma_{\Delta}^{\mathrm{em}}+\Gamma_{\Delta}^{\mathrm{str}}\right)=0.55 \ldots 0.65 \%$. The predictions for the generalized spin polarizabilities and other moments of the spin structure functions are thus parameter-free. It is also important to stress that in the covariant scheme employed here one has more loop diagrams at leading order as compared to the heavy baryon approach (cf. Fig. 2 in [8]). In that approach, the "missing" graphs only appear at fourth order due to the additional counting in the inverse baryon mass. Here, we have to deal with 14 different topologies as shown in Fig. 2. None of them involves the leading, dimension-two $\Delta N \gamma-$ vertices, such contributions only start at $\mathcal{O}\left(\varepsilon^{4}\right)$. Still, the algebra to evaluate the diagrams shown in Fig. 2 is non-trivial. In particular the box-type diagrams which three spin-3/2 propagators generate a large number of terms, as the spin-3/2 field propagator is given by

$$
S^{\mu \nu}=\frac{\not p+m_{\Delta}}{p^{2}-m_{\Delta}^{2}}\left(-g^{\mu \nu}+\frac{1}{3} \gamma^{\mu} \gamma^{\nu}+\frac{1}{3 m_{\Delta}}\left(\gamma^{\mu} p^{\nu}-\gamma^{\nu} p^{\mu}\right)+\frac{2}{3 m_{\Delta}^{2}} p^{\mu} p^{\nu}\right) .
$$

For example, the box diagram (right-most graph in the upper row of Fig. 2) has $5^{3}=125$ times more terms than the corresponding pion-nucleon box graph. Therefore, we have developed our own algebraic program that combines FORM [15] and Mathematica to calculate the tree and the loop diagrams. The code is able to reduce tensor integrals of any rank in the relativistic and the heavy baryon formalism. 
FORM is used to reduce the pertinent tensor integrals with higher powers of propagators and shifted dimensions while Mathematica is utilized to perform the standard Passarino-Veltman reduction [16] (if required). In particular, the program allows for an easy heavy mass reduction of any given relativistic formulation.

We have calculated the spin-polarizabilities $\gamma_{0}\left(Q^{2}\right), \delta_{0}\left(Q^{2}\right)$, the generalized GDH integral $I_{A}\left(Q^{2}\right)$ and also the first moment $\Gamma_{1}\left(Q^{2}\right)$ for the neutron and the proton. The resulting expressions for the loop contributions are very lengthy and will not be given here explicitly\#5. However, we mention that our framework allows to take the heavy baryon limit in which the nucleon and the delta are considered as heavy, static sources keeping the mass splitting fixed. Indeed, we recover the heavy baryon results of Ref. [8]. For better comparison, we give here the explicit Born terms corresponding to Fig. [1]

$$
\begin{aligned}
S_{1}^{\Delta-\operatorname{Born}}\left(\nu, Q^{2}\right) & =\frac{2 e^{2} b_{1}^{2}}{9 m_{\Delta}^{2}} \cdot \frac{1}{\left(m_{\Delta}^{2}-m_{N}^{2}+Q^{2}\right)^{2}-4 m_{N}^{2} \nu^{2}}\left[2 m_{N}^{2} \nu^{2}\left(m_{N}^{2}-3\left(Q^{2}+m_{\Delta}^{2}\right)\right)\right. \\
& \left.+Q^{2}\left(m_{\Delta}^{2}-m_{N}^{2}+Q^{2}\right)\left(3 m_{\Delta}^{2}-2 m_{N}^{2}+2 Q^{2}-2 m_{N} m_{\Delta}\right)\right], \\
S_{2}^{\Delta-\operatorname{Born}}\left(\nu, Q^{2}\right) & =-\frac{4 e^{2} b_{1}^{2} m_{N}^{2} \nu}{9 m_{\Delta}^{2}} \frac{\left(m_{N}+m_{\Delta}\right)\left(m_{N}^{2}+Q^{2}-2 m_{N} m_{\Delta}\right)}{\left(m_{\Delta}^{2}-m_{N}^{2}+Q^{2}\right)^{2}-4 m_{N}^{2} \nu^{2}} .
\end{aligned}
$$

\section{Results and discussion}

For obtaining numerical results, we use the following set of parameters: $g_{A}=1.27, F_{\pi}=92.21 \mathrm{MeV}$, $M_{\pi}=138.04 \mathrm{MeV}, m_{N}=938.9 \mathrm{MeV}, \kappa_{v}=3.706, \kappa_{s}=-0.120, m_{\Delta}=1232 \mathrm{MeV}$ (in the appendix, we also discuss some results obtained using the S-matrix pole mass as determined e.g. in pion-nucleon scattering). For the $\Delta$ couplings, we obtain from Eqs. (19/20)

$$
h_{A}=1.43 \pm 0.02, \quad b_{1}=-(4.98 \pm 0.27) / m_{N} .
$$

For comparison, the corresponding large- $N_{C}$ relations yield $h_{A}=\left(3 g_{A}\right) /(2 \sqrt{2})=1.35$, and $b_{1}=$ $-3\left(1+\kappa_{p}-\kappa_{n}\right) /\left(2 \sqrt{2} m_{N}\right)=-5.0 / m_{N}$, which are consistent with the empirical values. Note that we take the sign of $h_{A}$ and $b_{1}$ to be consistent with the large- $N_{C}$ relations, as the formulae for the corresponding width are quadratic in these couplings. We will generate theoretical errors by varying these couplings within the ranges given above. Uncertainties due to neglected higher orders will not be considered.

First, we consider the forward and the longitudinal-transverse spin-polarizabilities at the photon point, $\gamma_{0}(0)$ and $\delta_{0}(0)$, respectively. We find using the central values of the input parameters

$$
\begin{aligned}
\gamma_{0}^{p} & =2.07_{q^{3}}-3.65_{\epsilon^{3}, \text { tree }}-0.16_{\epsilon^{3}, \text { loop }}=-1.74[ \pm 0.40], \\
\gamma_{0}^{n} & =3.06_{q^{3}}-3.65_{\epsilon^{3}, \text { tree }}-0.18_{\epsilon^{3}, \text { loop }}=-0.77[ \pm 0.40], \\
\delta_{0}^{p} & =1.54_{q^{3}}-0.36_{\epsilon^{3}, \text { tree }}+1.22_{\epsilon^{3}, \text { loop }}=2.40[ \pm 0.01], \\
\delta_{0}^{n} & =2.41_{q^{3}}-0.36_{\epsilon^{3}, \text { tree }}+0.33_{\epsilon^{3}, \text { loop }}=2.38[ \pm 0.03],
\end{aligned}
$$

in units of $10^{-4} \mathrm{fm}^{4}$. The first term refers to the third order pion-nucleon loop result, whereas the second and third term are the delta tree and loop corrections at third order in the SSE. In brackets, we give the results due to the variation of $h_{A}$ and $b_{1}$ within the bounds given above. We do not attempt here to estimate the error stemming from the fourth (and higher) order terms - this issue will be dealt with in the future when we present the results of the complete one-loop analysis. As

\footnotetext{
\#5 They can be made available as a Mathematica notebook upon request from Hermann Krebs.
} 

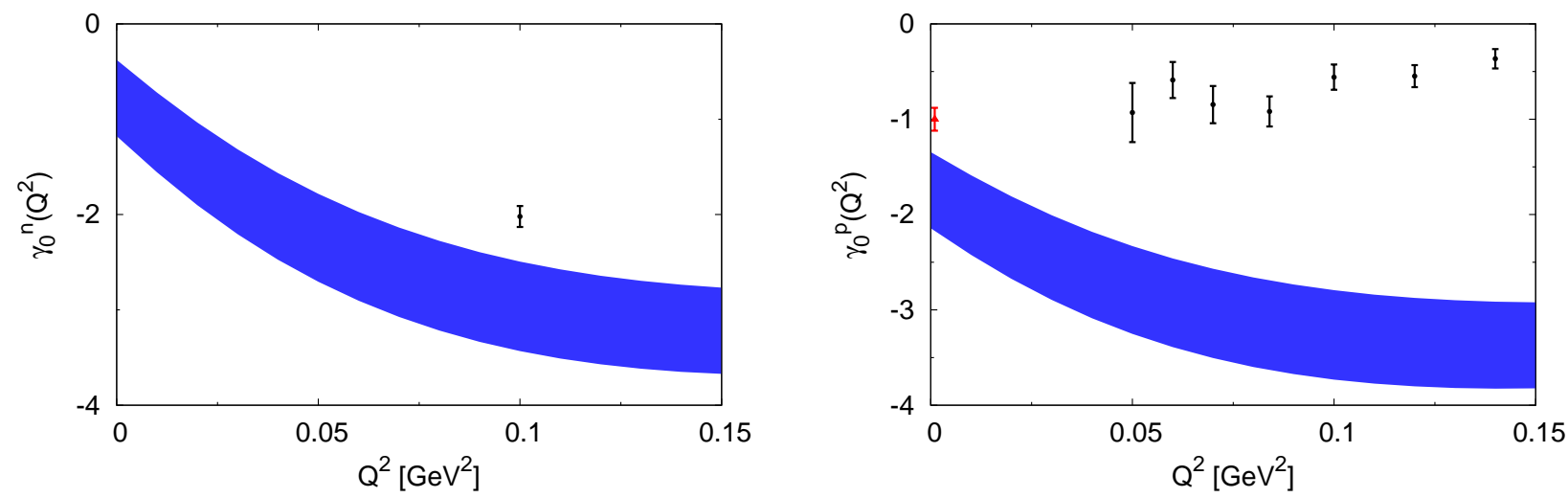

Figure 3: Forward spin polarizability in units of $10^{-4} \mathrm{fm}^{4}$ at finite photon virtuality for the neutron (left) and the proton (right). Neutron data: Ref. [3] and proton data from Ref. [17] $\left(Q^{2}=0\right)$ and Ref. [4] $\left(Q^{2}>0\right)$. Only statistical errors are shown.

already found in Ref. [10, the corrections from tree-level delta graphs are large in the forward spinpolarizabilities whereas the delta loop corrections for $\gamma_{0}^{n, p}$ are very small\#6 This is different for the transverse-longitudinal polarizabilities, where the tree contributions are suppressed (as it was also found in the heavy baryon calculation of [8]). We note that the parameter-free prediction for $\gamma_{0}^{p}$ agrees within $1.5 \sigma$ with the empirical number, $\gamma_{0}^{p}=-1.00 \pm 0.08 \pm 0.12$ [17]. We note that the latter number is obtained using the well-known sum rule for $\gamma_{0}$ in terms of the measured difference of the photon-proton cross sections with helicity $1 / 2$ and $3 / 2$ for photon energies between 200 and $1800 \mathrm{MeV}$ combined with the MAID2003 prediction for the region between the threshold and $200 \mathrm{MeV}$. This is a clear improvement as compared to earlier calculations employing either HBCHPT with explicit deltas or the covariant $\mathcal{O}\left(q^{4}\right)$ calculation adding tree-level $\Delta$-contributions. Of course, before one can claim success, one must consistently evaluate the fourth order contributions from nucleon and delta intermediate states. We also note the marked difference in the delta-loop contribution to $\delta_{0}^{p}$. While in the heavy baryon scheme this contribution is small, it is sizeable in our relativistic approach. This can be largely traced back to the box diagram (the right-most diagram in the upper row of Fig. 2). As $m_{N}$ and $m_{\Delta}$ (with the splitting fixed) tend to infinity, the contribution from this diagram vanishes, whereas its value is $1.32 \cdot 10^{-4} \mathrm{fm}^{4}$ in our case. We have analyzed the $1 / m_{N}$-expansion\#7 of the box diagram. We find that it only starts to contribute at $\mathcal{O}\left(1 / m_{N}^{2}\right)$ and that the convergence of the series is very slow. The effect is much more dramatic for the proton than for the neutron due to a much larger prefactor. This shows that the heavy baryon expansion does not provide a good approximation to the covariant result for this observable.

Next, we consider the various observables at finite photon virtuality. In Fig. 3, we show the neutron (left panel) and proton (right panel) forward spin polarizabilities for virtualities $Q^{2} \leq 0.15 \mathrm{GeV}^{2}$. For the neutron, there is only one data point at $Q^{2}=0.1 \mathrm{GeV}^{2}$, which lies slightly above the predictions. The trend of the proton data is not recovered, the discrepancy between the chiral prediction and the data grows with increasing photon virtuality. This is also reflected in the deviations of the isoscalar and isovector combinations at $Q^{2}=0.1 \mathrm{GeV}^{2}$ given in Ref. [18. To get more insights into these trends, we display the same decomposition for $\gamma_{0}^{p, n}\left(Q^{2}\right)$ at $Q^{2}=0.1 \mathrm{GeV}^{2}$ as given at the photon point in

\footnotetext{
${ }^{\# 6}$ In this work we use dimensional and not infrared regularization as in Ref. 10. For this reason only qualitative comparison is possible between our results and that of [10].

${ }^{\# 7}$ The $1 / m_{N}$ expansion with fixed $\Delta=m_{\Delta}-m_{N}$ leads to the usual heavy baryon SSE.
} 

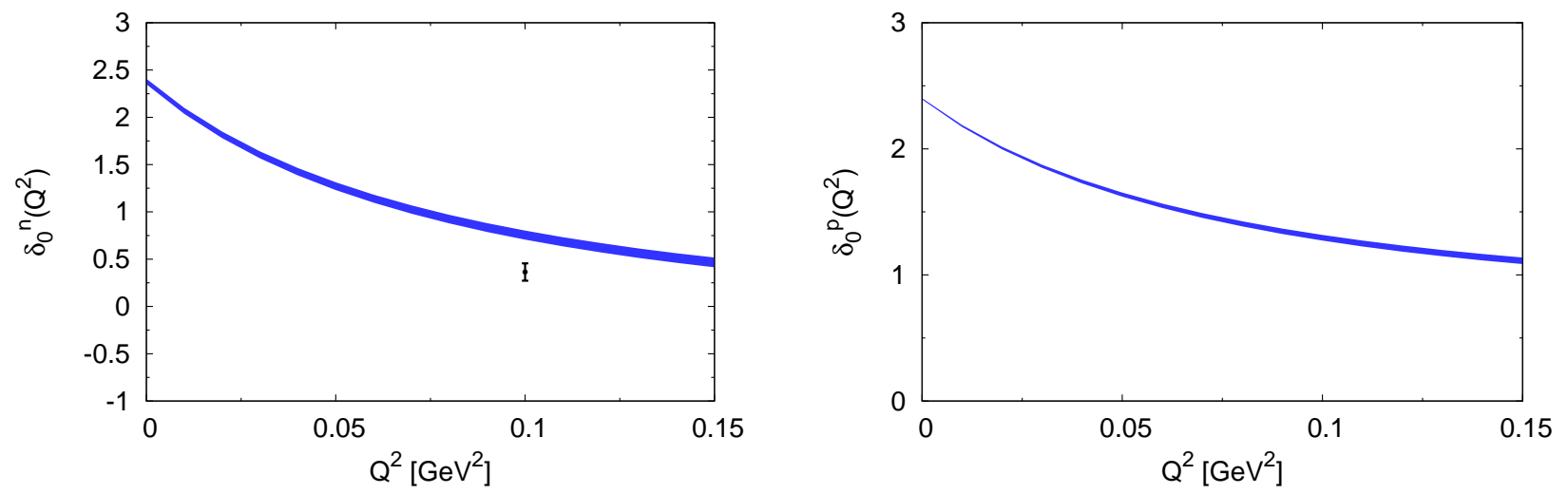

Figure 4: Longitudinal-transverse spin polarizability in units of $10^{-4} \mathrm{fm}^{4}$ at finite photon virtuality for the neutron (left) and the proton (right). Neutron data: Ref. [3]. Only statistical errors are shown.

Eq. (24):

$$
\begin{aligned}
& \gamma_{0}^{p}=1.17_{q^{3}}-4.29_{\epsilon^{3}, \text { tree }}-0.13_{\epsilon^{3}, \text { loop }}=-3.25[ \pm 0.48], \\
& \gamma_{0}^{n}=1.49_{q^{3}}-4.29_{\epsilon^{3}, \text { tree }}-0.15_{\epsilon^{3}, \text { loop }}=-2.95[ \pm 0.48] .
\end{aligned}
$$

We see that the decrease of $\gamma_{0}^{p, n}\left(Q^{2}\right)$ is a combined effect of a decreasing positive contribution of the pion-nucleon loops and an increase in magnitude of negative contribution from the $\Delta$ tree graphs. Again, a complete fourth calculations is required to settle the issue. Given that the prediction for $\gamma_{0}^{p}$ at the photon point is already close to experiment, one may hope that such a fourth order calculation would provide a fine test of the chiral QCD dynamics in view of the upcoming data at low photon virtualities from Jefferson Lab (down to $Q^{2} \approx 0.01 \mathrm{GeV}^{2}$ ). It is also interesting to confront our predictions with the isospin separated forward spin-polarizabilities of Ref. [18. At $Q^{2}=0.1 \mathrm{GeV}^{2}$, these authors find $\gamma_{0}^{p-n}=1.53$ and $\gamma_{0}^{p+n}=-2.51$. This should be compared with our predictions of $\gamma_{0}^{p-n}=-0.30$ and $\gamma_{0}^{p+n}=-6.20$ for the central values (all in canonical units). The third order SSE calculation disagrees markedly from the experimental values. As stressed before, a complete $\mathcal{O}\left(\varepsilon^{4}\right)$ calculation is called for.

The $Q^{2}$-dependence of the transverse-longitudinal spin polarizability is shown in Fig. 4. In contrast to the IR calculation of Ref. [10, it is a monotonically decreasing function of $Q^{2}$ for both the neutron and the proton. This is a generic feature of using dimensional instead of infrared regularization. Still, the only empirical value of $\delta_{0}^{n}\left(Q^{2}=0.1 \mathrm{GeV}^{2}\right)$ is slightly missed by the chiral prediction, but again a clear improvement as compared to earlier calculations is achieved. We remark again that the uncertainty shown here does not involve the effects of higher orders not considered here. The decrease of $\delta_{0}^{p, n}\left(Q^{2}\right)$ is mostly due to the pion-nucleon loop graphs, cf. Eq. (24)

$$
\begin{aligned}
\delta_{0}^{p}=0.59_{q^{3}}-0.53_{\epsilon^{3}, \text { tree }}+1.23_{\epsilon^{3}, \text { loop }}= & 1.29[ \pm 0.03] \\
\delta_{0}^{n}=0.95_{q^{3}}-0.53_{\epsilon^{3}, \text { tree }}+0.33_{\epsilon^{3}, \text { loop }}= & 0.75[ \pm 0.05] .
\end{aligned}
$$

The $Q^{2}$-dependence of the generalized GDH sum rule $I_{A}\left(Q^{2}\right)$ is shown in Fig. 5. Here, we find a clear difference to the data point at $Q^{2}=0.1 \mathrm{GeV}^{2}$, whereas the phenomenological inclusion of the tree level $\Delta$-terms produced a broad band that was consistent with this datum. It remains to be seen how the complete one-loop calculation will do, as we know that there are sizeable $\mathcal{O}\left(q^{4}\right)$ pion-nucleon loop corrections. 

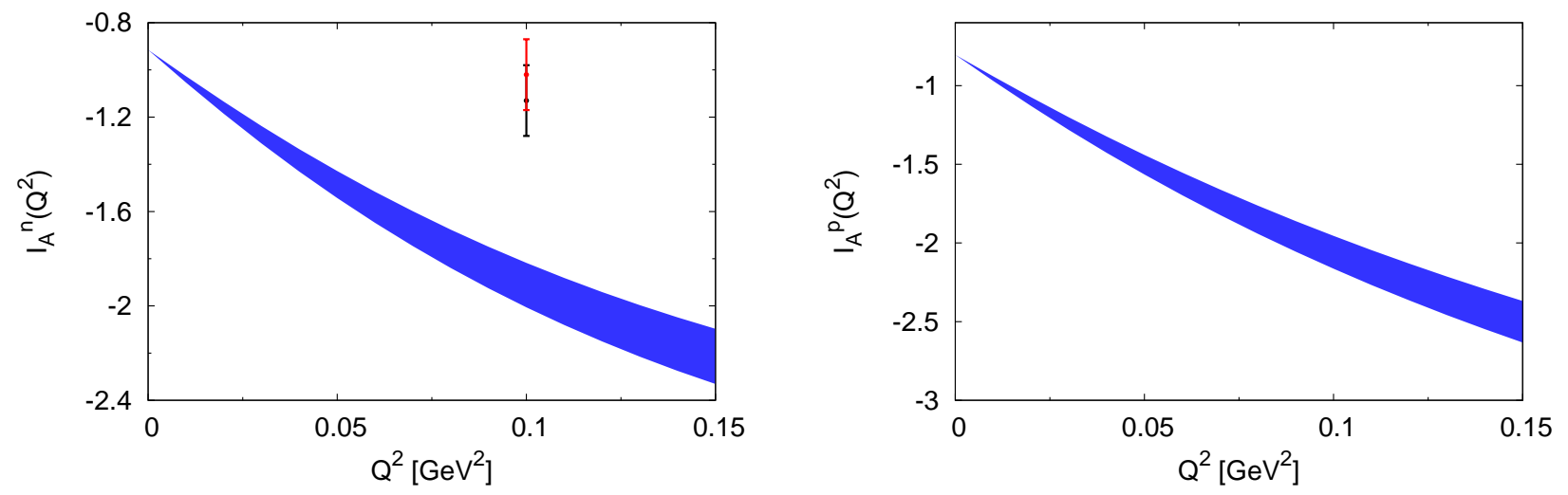

Figure 5: Generalized GDH integral $I_{A}$ for the neutron (left) and the proton (right). Neutron data: Ref. [2]. The two data points at the same value of $Q^{2}$ refer to different extraction methods as described in [2]. Only the dominant systematic errors are shown.

Finally, the $Q^{2}$-dependence of the first moment $\Gamma_{1}\left(Q^{2}\right)$ for the proton and the isovector combination $\Gamma_{1}^{(p-n)}\left(Q^{2}\right)$ are displayed in Fig. 6, While the $\mathcal{O}\left(\varepsilon^{3}\right)$ contributions slightly improve the chiral prediction for the proton, more curvature from the pion-nucleon and pion-delta loop graphs at fourth order is required. This again points towards the necessity of performing such a complete fourth order calculations within the framework outlined here. However, we note that the third order calculation already describes the admittedly relatively imprecise data for the isovector combination $\Gamma_{1}^{(p-n)}\left(Q^{2}\right)$ taken from Ref. [18]. Therefore, in this combination the fourth order corrections should largely cancel, which was found to be the case in the heavy baryon approach [12] but not in the infrared regularized covariant calculation [9].
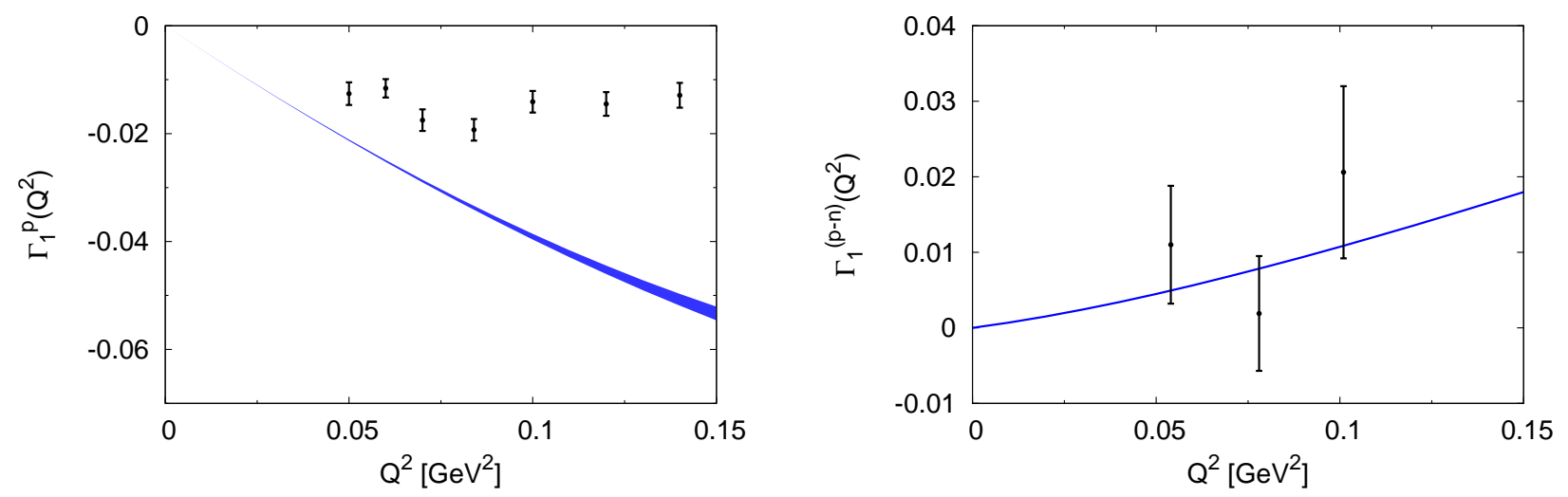

Figure 6: First moment of the integral $I_{1}\left(Q^{2}\right)$ for the the proton (left) and the isovector nucleon $(p-n)$ (right). The proton data are from Ref. [4] and the isovector data from Ref. [18]. Only statistical errors are shown. 


\section{$5 \quad$ Summary and outlook}

We have presented a calculation of the nucleon spin structure at low energies in the framework of a covariant formulation of baryon chiral effective field theory with explicit spin-3/2 degrees of freedom. We have included all terms up-to-and-including $\mathcal{O}\left(\varepsilon^{3}\right)$ in the small scale expansion. At this order, one has contributions from delta tree diagrams supplemented by the leading pion-nucleon and pion-delta loop graphs. Having fixed the LECs from the strong and the electromagnetic width of the $\Delta$-resonance, we can make parameter-free predictions. This is particularly useful in view of the upcoming precision data from Jefferson Lab at low photon virtualities. The main results of this investigation can be summarized as follows:

i) We find an improved description of the forward spin polarizability $\gamma_{0}$ for the neutron and the proton. In particular, the value of $\gamma_{0}^{p}(0)$ is consistent with the determination from the GDH collaboration. However, the $Q^{2}$-dependence of the $\gamma_{0}^{p}\left(Q^{2}\right)$ is not consistent with the data, with the discrepancy increasing with larger photon virtuality.

ii) For the longitudinal-transverse spin polarizability, we find an improved description as compared to earlier calculations. Still, the experimental value of $\delta_{0}^{n}\left(Q^{2}=0.1 \mathrm{GeV}^{2}\right)$ is slightly larger in magnitude than the chiral prediction.

iii) The generalized GDH integral $I_{A}\left(Q^{2}\right)$ shows a faster fall-off with increasing photon virtuality as indicated by the data on the neutron. $\pi N$ and $\pi \Delta$ loops at fourth order are expected to supply the necessary curvature.

iv) Similar statements can be made for the first moment $\Gamma_{1}\left(Q^{2}\right)$, where the approximate $Q^{2}$ independence of the proton data (for small values of $Q^{2}$ ) is not captured by the third order

calculation. However, we find that the prediction for the isovector combination $\Gamma_{1}^{(p-n)}\left(Q^{2}\right)$ is in agreement with the few existing but not very precise data.

All this points towards the necessity of performing a complete one-loop calculation [19]. However, we would like to stress that what was considered here are just the leading contributions based on a covariant effective Lagrangian with explicit deltas - as such, most of the results can be considered quite encouraging. In view of the upcoming Jefferson Lab data at very small photon virtualities, one can finally hope to test the chiral QCD dynamics related to the nucleon spin structure with sufficient precision.

\section{Acknowledgments}

We thank Marina Dorati for collaboration during the early stage of this investigation and Jianping Chen for strong encouragement to write up these results. Work supported in part by DFG (SFB/TR 16, "Subnuclear Structure of Matter"), by the European Community-Research Infrastructure Integrating Activity "Study of Strongly Interacting Matter" (acronym HadronPhysics3, Grant Agreement n. 283286) under the Seventh Framework Programme of EU, and and ERC project 259218 NUCLEAREFT. 


\section{A Modifying the $\Delta$-resonance input}

So far, we have used the Breit-Wigner mass for the $\Delta, m_{\Delta}=1232 \mathrm{MeV}$ and the corresponding width, as it was done e.g. in Ref. [10]. However, one might alternatively use the parameters from the S-matrix pole deduced from pion-nucleon scattering and pion photoproduction, which are currently listed as

$$
m_{\Delta}=1210 \mathrm{MeV}, \quad-2 \operatorname{Im} \Sigma_{\Delta}=\Gamma_{\Delta}^{\mathrm{str}}+\Gamma_{\Delta}^{\mathrm{em}}=(100 \pm 2) \mathrm{MeV} .
$$

Using these values, the couplings $h_{A}$ and $b_{1}$ change to

$$
h_{A}=1.51 \pm 0.02, \quad b_{1}=-(5.10 \pm 0.27) / m_{N} .
$$

These are consistent within uncertainties with the earlier values, cf. Eq. (23), but we note that the central value for $b_{1}$ has increased by about $3 \%$. Using this new input, we have repeated the calculation. Here, we only show the modified results for the spin-polarizabilities $\gamma_{0}$ and $\delta_{0}$ at the photon point and one finite photon virtuality. We find at $Q^{2}=0$ :

$$
\begin{aligned}
\gamma_{0}^{p}=2.07_{q^{3}}-4.52_{\epsilon^{3}, \text { tree }}-0.22_{\epsilon^{3}, \text { loop }}=-2.67[ \pm 0.49], \\
\gamma_{0}^{n}=3.06_{q^{3}}-4.52_{\epsilon^{3}, \text { tree }}-0.23_{\epsilon^{3}, \text { loop }}=-1.69[ \pm 0.48], \\
\delta_{0}^{p}=1.54_{q^{3}}-0.42_{\epsilon^{3}, \text { tree }}+1.40_{\epsilon^{3} \text { loop }}=2.52[ \pm 0.01], \\
\delta_{0}^{n}=2.41_{q^{3}}-0.42_{\epsilon^{3}, \text { tree }}+0.37_{\epsilon^{3}, \text { loop }}=2.36[ \pm 0.04] .
\end{aligned}
$$

While there are only small changes in the delta-loop contributions, the delta-tree terms are markedly enlarged, which is in particular relevant for $\gamma_{0}$. This increase is approximately to one third due to the increased value of $b_{1}$ and to two thirds related to the smaller $\Delta$-mass in denominator, cf. Eq. (22) (see also Eq. (39) in Ref. [8]). Also consistent with the heavy baryon results [8], the corrections to $\delta_{0}$ are less significant. These trends persist at finite photon virtualities. At $Q^{2}=0.1 \mathrm{GeV}^{2}$, we find

$$
\begin{aligned}
& \gamma_{0}^{p}=1.17_{q^{3}}-5.52_{\epsilon^{3}, \text { tree }}-0.17_{\epsilon^{3}, \text { loop }}=-4.52[ \pm 0.60], \\
& \gamma_{0}^{n}=1.49_{q^{3}}-5.52_{\epsilon^{3}, \text { tree }}-0.20_{\epsilon^{3}, \text { loop }}=-4.23[ \pm 0.60], \\
& \delta_{0}^{p}=0.59_{q^{3}}-0.65_{\epsilon^{3}, \text { tree }}+1.42_{\epsilon^{3}, \text { loop }}=1.36[ \pm 0.03], \\
& \delta_{0}^{n}=0.95_{q^{3}}-0.65_{\epsilon^{3}, \text { tree }}+0.37_{\epsilon^{3}, \text { loop }}=0.67[ \pm 0.06] .
\end{aligned}
$$

These data show the same trends as at the photon point, cf. Eqs. (25126). As we are only considering the leading delta tree and loop graphs here, we expect that some of the uncertainty induced by the values for $m_{\Delta}, \Gamma_{\Delta}$ will be reduced when the subleading $\mathcal{O}\left(\varepsilon^{4}\right)$ corrections are included.

\section{References}

[1] S. E. Kuhn, J.-P. Chen and E. Leader, Prog. Part. Nucl. Phys. 63 (2009) 1 [arXiv:0812.3535 [hep-ph]].

[2] M. Amarian, L. Auerbach, T. Averett, J. Berthot, P. Bertin, W. Bertozzi, T. Black and E. Brash et al., Phys. Rev. Lett. 89, 242301 (2002) nucl-ex/0205020].

[3] M. Amarian et al. [Jefferson Lab E94010 Collaboration], Phys. Rev. Lett. 93 (2004) 152301 nucl-ex/0406005.

[4] Y. Prok et al. [CLAS Collaboration], Phys. Lett. B 672 (2009) 12 [arXiv:0802.2232 [nucl-ex]]. 
[5] J. P. Chen, Int. J. Mod. Phys. E 19 (2010) 1893 [arXiv:1001.3898 [nucl-ex]].

[6] V. Sulkosky, "An Overview of Longitudinal Spin Structure Measurements from JLab," talk at Chiral Dynamics 2012, Jefferson Lab., August 2012.

[7] V. Bernard, Prog. Part. Nucl. Phys. 60, 82 (2008) [arXiv:0706.0312 [hep-ph]].

[8] C. W. Kao, T. Spitzenberg and M. Vanderhaeghen, Phys. Rev. D 67, 016001 (2003) arXiv:hep-ph/0209241.

[9] V. Bernard, T. R. Hemmert and U.-G. Meißner, Phys. Lett. B 545, 105 (2002) arXiv:hep-ph/0203167.

[10] V. Bernard, T. R. Hemmert and U.-G. Meißner, Phys. Rev. D 67, 076008 (2003) arXiv:hep-ph/0212033.

[11] J. Edelmann, N. Kaiser, G. Piller and W. Weise, Nucl. Phys. A 641 (1998) 119 nucl-th/9806096.

[12] X. D. Ji and J. Osborne, J. Phys. G 27, 127 (2001) arXiv:hep-ph/9905410.

[13] T. R. Hemmert, B. R. Holstein and J. Kambor, J. Phys. G 24, 1831 (1998) arXiv:hep-ph/9712496.

[14] V. Bernard, T. R. Hemmert and U.-G. Meißner, Phys. Lett. B 622 (2005) 141 arXiv:hep-lat/0503022.

[15] J. A. M. Vermaseren, arXiv:math-ph/0010025.

[16] G. Passarino and M. J. G. Veltman, Nucl. Phys. B 160 (1979) 151.

[17] H. Dutz et al. [GDH Collaboration], Phys. Rev. Lett. 91 (2003) 192001.

[18] A. Deur, P. Bosted, V. Burkert, D. Crabb, V. Dharmawardane, G. E. Dodge, T. A. Forest and K. A. Griffioen et al., Phys. Rev. D 78 (2008) 032001 [arXiv:0802.3198 [nucl-ex]].

[19] V. Bernard, E. Epelbaum, H. Krebs and U.-G. Meißner, in preparation. 